\title{
Diferencias entre los estilos de amar que tienen hombres y mujeres y sus reacciones de Estrés Postraumático tras la ruptura de su relación
}

\author{
Man Cheung Chung* \\ Steven Farmer** \\ Keren Grant ${ }^{\star *}$ \\ Rebecca Newton** \\ Sally Payne** \\ Melissa Perry ${ }^{\star \star}$ \\ Jenny Saunders** \\ Charlotte Smith** \\ Nina Stone** \\ * Universidad de Plymouth \\ ** Universidad de Wolverhampton \\ REINO UNIDO
}

\begin{abstract}
RESUMEN - Este estudio pretendía explorar 1) las diferencias existentes entre el sexo femenino y el masculino a la hora de manifestar síntomas de estrés postraumático tras la ruptura de una relación sentimental, 2) las diferencias en el estilo de amar que presenta cada sexo durante la relación y, 3) la interacción entre el sexo, el estrés traumático y las distintas maneras de expresar el amor. Valoramos la posibilidad de que ambos sexos se diferenciaran en cuanto a los síntomas de estrés postraumático tras la ruptura de una relación así como en los estilos de amar adoptados y asimismo, consideramos la posibilidad de que existiera una interacción significativa entre el sexo, el estrés traumático y los estilos de amar. Un total de 25 hombres y 40 mujeres participaron en el estudio y fueron entrevistados con la Escala de Impacto de Acontecimientos Vitales (IES), el Cuestionario de Salud General (GHQ) y la Escala de Actitud ante el amor (LAS). Los resultados no mostraron diferencias significativas entre ambos sexos en lo que al impacto que provocaba la ruptura se refiere, sin embargo, las mujeres padecían mayor disfunción social y depresión que los hombres. Los resultados también mostraron que existían diferencias en los estilos de amor "Eros" y "Agape". A pesar de ello, no se obtuvieron resultados significativos en las relaciones existentes entre el sexo, la gravedad del estrés traumático y los estilos de amar.
\end{abstract}




\section{Introducción}

Se han llevado a cabo innumerables investigaciones con el fin de entender la relación que existe entre el estrés postraumático y otros tipos de cambios como el tecnológico (e.g. Weisaeth 1995), los desastres naturales (e.g. Gerrity y Steinglass 1995), y los accidentes (e.g. Mitchell 1997). Recientemente, los investigadores han intentado ampliar el concepto de estrés postraumático a un área que va más allá de los límites propios de estos desastres, como es el área de la ruptura de relaciones. Glass y Wright (1997) y Lusterman (1995) han establecido una relación entre las reacciones de estres postraumático y problemas de pareja tal como el de la infidelidad. Según estos autores, aquellos miembros de la pareja que han sido traicionados podrían volver a experimentar el descubrimiento traumático de la infidelidad en sueños, a través "flashbacks" y recuerdos intrusos. Normalmente recordar la fecha en la que se descubrió que su pareja tuvo una aventura, las canciones de amor y los espectáculos en televisión son los desencadenantes de este tipo de reacciones. Los cónyuges que han sido traicionados también pueden mostrar actitudes de rechazo aparentando indiferencia ante la infidelidad y menos interés por los demás y el mundo exterior. Pueden hacer ver que se encuentran muy relajados ante la infidelidad y no expresar sentimientos al respecto. También es posible que experimenten insomnio, agitación, irritabilidad, ira y otro tipo de reacciones más llamativas. A pesar de todo, prácticamente no existen investigaciones sobre el origen del estrés postraumático en este contexto. Uno de los objetivos de este estudio es el de arrojar alguna luz sobre el origen del estrés postraumático tras la ruptura de una relación sentimental.
Aunque aún se desconoce el origen del estrés postraumático que se produce cuando finaliza una relación, según las investigaciones realizadas en este terreno, estas rupturas constituyen un proceso muy estresante y traumático (Bloom, Asher y White 1978, Menaghan y Lieberman 1986, Stroebe y Stroebe 1986). Se ha descubierto que aquellas personas que han pasado por la experiencia de una ruptura sentimental, tienen más problemas emocionales, tales como la soledad y la depresión, que aquellas otras que no han vivido esta experiencia (Sprecher 1994). También pueden manifestar reacciones de tristeza (Lagrand 1988) con síntomas de psicológicos y físicos negativos como el insomnio o las ideas suicidas, que pueden durar más de seis meses. (Fink, Russell, Engel, Harkness, Pederson y Harvey 1991) La ruptura de una relación también puede dar lugar al aumento del consumo de alcohol, o a la pérdida de peso (Mastekaasa 1997, 1994) e incluso a enfermedades físicas, (Williams y Siegel 1989), siendo la depresión más frecuente al principio de la ruptura. (Mearns 1991). Las mujeres divorciadas tienden a presentar síntomas depresivos más graves que las mujeres casadas (Lorenz, Simons y Chao 1996). Suelen culparse por la ruptura, se sienten indefensas ante su futuro y se convierten en personas aprensivas. También muestran cierta tendencia a recordar experiencias más o menos intensas y desagradables de la relación que ha terminado (Harvey, Flanary y Morgan 1986). Del mismo modo, pueden presentar otras respuestas emocionales que incluyen sentimientos de culpabilidad, inseguridad, miedo, ira, odio, rechazo, autocompasión, vacío, disminución de la confianza en sí mismas y sensación de perdida (Lagrand 1988).

Los hombres y las mujeres experimentan angustia tras la ruptura de una relación de 
forma diferente aunque los estudios que hay al respecto presentan distintos resultados. Por un lado, se descubrió que, al terminar una relación, los hombres se sentían, en gran medida, deprimidos, solos, infelices y menos libres que las mujeres. (Hill, Rubin y Peplau 1976, Rubin, Peplau y Hill 1981). Por otro lado y, según otro estudio, las mujeres tendían a padecer depresiones más graves que los hombres (Mearns 1991). Otro estudio matizaba que, comparadas con los hombres, las mujeres se mostraban más angustiadas ante la ruptura, le otorgaban más importancia y tendían a mostrar una actitud de rechazo (Smith y Cohen 1993). Sin embargo, en algunas investigaciones no se descubrieron diferencias en lo que a la angustia experimentada tras la ruptura de la relación se refiere (Frazier y Cook 1993, Helgeson 1994, Hortacsu y Karanci 1987, Simpson 1987).

Los distintos estilos o actitudes ante el amor que las personas mantuvieron durante la relación podrían ser responsables, de alguna manera, de la angustia psicológica que padecen tras la ruptura de la relación. Por ejemplo, si una persona adopta una actitud de jugueteo, la angustia psicológica tras la ruptura de la relación podría no ser tan grave como la de una persona que tiene una actitud posesiva. Sin embargo, las diferentes investigaciones que se han llevado a cabo han aportado muy poca información sobre las relaciones entre los distintas formas de amar y la angustia psicológica que surge tras la ruptura de una relación. La información existente sobre la relación entre los distintos tipos de amor y el estrés postraumático tras la ruptura de una relación, es incluso menos ilustrativa. Por tanto, otro de los objetivos de este estudio es aportar más datos sobre este tipo de conexiones.

Actualmente, se está empezando a prestar más atención a las diferentes formas de amar las cuales pueden ser clasificadas en seis tipos: Eros, Ludus, Storge, Pragma, Mania y Ágape (Lee 1973, 1988). Los amantes "Eros" son amantes apasionados que saben exactamente el tipo de cualidades físicas que buscan en el otro y en consecuencia, tienden a relacionarse con personas que se adaptan a las características físicas deseadas. Suelen ser muy seguros de sí mismos y su autoestima es alta. También tienden a centrarse en sus parejas de una manera exclusiva e intensa sin caer en la posesión y los celos.

Los amantes "Ludus" son jugadores del amor basándolo en el divertimento mutuo más que en un intento serio. Estos amantes tienden a divertirse jugando con diferentes parejas al mismo tiempo, lo que, evidentemente evita que se impliquen seriamente con una persona en concreto. Además, no suelen mostrar preferencias en cuanto a la apariencia física por ninguna de sus parejas. La actitud que mantienen los amantes "Ludus" no implica que quieran herir a otras personas, en realidad su intención es la de minimizar el daño que puedan causar a los demás estableciendo las reglas cuando comienza la relación. Simplemente, quieren disfrutar de la vida con gente diferente.

Los amantes "Storge" son amantes tranquilos y poco activos. Basan el amor en la amistad, en el compañerismo y en la seguridad, y sus parejas comparten con ellos actitudes y valores. Para los amantes "Storge", dichas actitudes y valores, son más importantes que el aspecto físico y la mera satisfacción sexual. En otras palabras, para este tipo de amantes los compromisos a largo plazo son más importantes que las diversiones pasajeras.

Los amantes "Pragma" se centran en el aspecto práctico de la relación. Suelen buscar parejas con quienes puedan llevar una 
vida satisfactoria y provechosa al mismo tiempo, es decir, no se centran en una ilusión pasajera. Aparentemente, los amantes "Pragma" comparten características con los amantes "Storge" aunque se diferencian en algunos aspectos, por ejemplo, los amantes "Pragma", suelen valorar determinadas "condiciones" como la seguridad económica o los antecedentes familiares antes de iniciar una relación.

Los amantes "Mania" son personas que consideran que el amor es doloroso a pesar de que lo anhelan. Este tipo de amantes suelen ser celosos, posesivos y obsesivos; no confían en la sinceridad y el compromiso de sus parejas, asimismo pueden padecer problemas de alimentación, de insomnio y sufrir altos (exaltación) y bajos (depresión). Normalmente, obligan a sus parejas a comprometerse en lugar de esperar a que sean ellas las que tomen esa decisión. Naturalmente, la relación suele terminar rompiéndose, confirmando de este modo el miedo que sienten.

Los amantes "Agape" se preocupan exclusivamente por el bienestar de sus parejas y anteponen los intereses del otro a los suyos propios. Estas personas comienzan una relación porque creen que tienen algo que ofrecer a su pareja sin buscar nada a cambio. Obtener placer sexual no es importante para ellos.

Se ha descubierto que existen varios factores que están relacionados con estos estilos de amar, entre los que se incluyen la religión (Hendrick y Hendrick 1987), la raza (Hendrick y Hendrick 1986, Huang 1999, Murstein, Merighi y Vyse 1991) así como determinadas diferencias entre hombres y mujeres, que son el tema principal de nuestro estudio. Los hombres resultan ser más eróticos y lúdicos mientras que las mujeres tienden a ser más pragmáticas y maníacas.
Ambos sexos muestran características parecidas respecto a la pasión que imprimen a las relaciones (Hendrick y Hendrick 1986). Por el contrario, Hong (1986) no encontró diferencia alguna entre hombres y mujeres en lo que a la visión pragmática del amor se refiere, aunque las mujeres eran considerablemente menos idealistas que los hombres en cuanto al amor romántico. En cambio, según las aportaciones de Hendrick y Hendrick, a los hombres latinos les gustaban más las actividades lúdicas y eran más fáciles de sorprender que las mujeres latinas (Leon, Parra, Cheng y Flores 1995). Sin embargo, se descubrió que las mujeres eran más eróticas y prácticas así como menos lúdicas y fáciles de comprender que los hombres (Rotenberg y Korol 1995). Nuestras investigaciones también demostraron que las mujeres, cuyas formas de amar eran "Agape" solían ser más estables y con menor tendencia a modificar sus tendencias. Los hombres que podían ser incluidos dentro de "Pragma" no solían experimentar tanto miedo. Los que pertenecían a "Eros", "Storge" y "Agape" eran más actuales. Los hombres "Eros" tenían menos variaciones en su percepción y los de "Mania" tenían más cambios. Los hombres de "Eros", "Mania" y "Agape" parecían ser los más complejos desde un punto de vista cognitivo. Aquellos otros que pertenecían al grupo de "Ludus" eran menos complicados y más flexibles (Hall, Hendrick y Hendrick 1991).

Este estudio tiene dos objetivos. En primer lugar, queremos aportar información sobre el origen de los síntomas de estrés postraumático tras la ruptura de una relación y valorar hasta que punto varía la manifestación de estos síntomas según el sexo de pertenencia. En segundo lugar, nuestro objetivo es el de valorar cómo varía la adopción de una determinada forma de amar dependiendo del sexo. En tercer lugar, pre- 
tendemos estudiar cómo interactúan las diferencias sexuales, el estrés traumático y los estilos de amar. Sobre la base de la bibliografía mencionada anteriormente, consideramos la posibilidad de que existieran diferencias sexuales en cuanto a la forma de manifestar los síntomas de estrés postraumático tras la ruptura de una relación así como en la adopción de una determinada forma de amar. Finalmente, consideramos que podría existir una interacción significativa entre el sexo, el estrés traumático y los estilos de amar.

\section{Método}

\section{Individuos}

La tabla I muestra los datos demográficos de las dos muestras $(M=25, F=40)$. La edad de ambos grupos era parecida y no existían diferencias en cuanto al estado civil de cada uno de los grupos $\left(\chi^{2}=0,78, n s\right)$. En el momento del estudio, el porcentaje de mujeres que mantenían una relación sentimental era considerablemente superior al de los hombres $\left(\chi^{2}=5,70, \mathrm{p}<0,01\right)$. Sin embargo, los dos grupos mostraron porcentajes parecidos en cuanto al periodo de tiempo transcurrido tras la ruptura $(\mathrm{t}=0,71$, $\mathrm{ns})$, la duración de las relaciones $(\mathrm{t}=-0,84$, ns) y la seriedad con la que las enfocaban $(\mathrm{t}=-0,79, \mathrm{~ns})$.

\section{Procedimiento}

Los voluntarios que participaron en este estudio obtuvieron información sobre él a través de carteles anunciadores que estuvieron expuestos en Residencias de estudiantes y en una Universidad inglesa durante apro-

Tabla I

Datos demográficos referidos al sexo

\begin{tabular}{|c|c|c|c|c|}
\hline \multirow[b]{2}{*}{ Items } & \multicolumn{2}{|c|}{$\begin{array}{c}\text { Hombres } \\
\mathrm{n}=25\end{array}$} & \multicolumn{2}{|c|}{$\begin{array}{c}\text { Mujeres } \\
\mathrm{n}=40\end{array}$} \\
\hline & $\mathrm{N}$ & $\%$ & $\mathrm{~N}$ & $\%$ \\
\hline \multicolumn{5}{|c|}{ Edad } \\
\hline \multicolumn{5}{|c|}{ Hombres: Media $=23,32, \mathrm{sd}=4,89$} \\
\hline \multicolumn{5}{|c|}{ Mujeres: Media = 23,43, sd = 9,18 } \\
\hline \multicolumn{5}{|c|}{ Estado civil } \\
\hline Casado & 2 & 8 & 3 & 7,5 \\
\hline Soltero & 22 & 88 & 33 & 82,5 \\
\hline Divorciado & 1 & 4 & 4 & 10 \\
\hline \multicolumn{5}{|c|}{ ¿Tienes alguna relación en este momento? } \\
\hline Sí & 10 & 40 & 28 & 70 \\
\hline No & 15 & 60 & 12 & 30 \\
\hline \multicolumn{5}{|c|}{ ¿Cuánto tiempo hace que tuvo lugar la ruptura (en meses)? } \\
\hline \multicolumn{5}{|c|}{ Hombres: Media $=6,62, \mathrm{sd}=5,31$} \\
\hline \multicolumn{5}{|c|}{ Mujeres: Media $=7,56, \mathrm{sd}=5,09$} \\
\hline \multicolumn{5}{|c|}{ ¿Cuánto tiempo duró la relación (en meses)? } \\
\hline \multicolumn{5}{|c|}{ Hombres: Media $=20,88, \mathrm{sd}=19,31$} \\
\hline \multicolumn{5}{|c|}{ Mujeres: Media $=29,01, \mathrm{sd}=45,62$} \\
\hline \multicolumn{5}{|c|}{ ¿Cómo de importante fue la relación? } \\
\hline Muy importante & 11 & 44 & 11 & 27,5 \\
\hline Importante & 6 & 24 & 15 & 37,5 \\
\hline Moderadamente importante & 7 & 28 & 13 & 32,5 \\
\hline Casual & 1 & 4 & 1 & 2,5 \\
\hline
\end{tabular}


ximadamente seis meses. Las personas que participaron habían experimentado la ruptura de una relación durante los 24 meses anteriores al estudio. Los voluntarios contactaron con los investigadores a través de la información que se adjuntaba en los carteles y las entrevistas se realizaron en sus propias casas. Se les pidió que rellenaran la escala que evalúa el impacto de los acontecimientos vitales, el Cuestionario de Salud General y la Escala de Actitudes ante el Amor.

\section{Formas de valoración}

La escala que evalúa el impacto de los acontecimientos vitales (IES) (Horowitz, Wilner y Álvarez 1979) es una escala de 15 items y cuatro puntuaciones $(0=$ no, nunca, 1 = en raras ocasiones, $3=$ algunas veces, $5=\mathrm{a}$ menudo), tratándose de un instrumento autoplicado que mide la presencia de recuerdos "intrusivos" así como las conductas de evitación frente al acontecimiento traumático. El cuestionario se distribuyó a dos muestras. Una de ellas estaba formada por personas que seguían una psicoterapia en régimen ambulatorio como consecuencia de un grave acontecimiento vital que les produjo un síndrome de estrés. La otra estaba formada por estudiantes de medicina que habían diseccionado su primer cadáver. Horowitz recomienda, utilizar a partir de la puntuación total, los puntos de corte de 8,5 o inferiores en las personas con síntomas más graves en la escala IES; de 8,6 a 19 para aquellos otros de tipo medio y de 19 o más para los de tipo más alto.

El Cuestionario de Salud General (GHQ28) (Goldberg y Hillier 1979) fue diseñado como un instrumento de estudio que pretende calcular la probabilidad de considerar a los individuos como "casos psiquiátricos" mediante entrevista. Las preguntas se clasificaron utilizando el método de puntuación de
0-0-1-1. El cuestionario consta de cuatro subescalas que incluyen los items "somáticos", de ansiedad, de disfunción social y de depresión. Si la puntuación total GHQ sobrepasa el punto de corte 4 recomendado, aumenta la probabilidad de tratarse de un "caso psiquiátrico".

La Escala de Actitud de Amor (LAS) (Hendrick y Hendrick 1990) fue diseñada con el fin de medir los estilos o actitudes de amar. Los 42 ítems de la LAS se puntuaron mediante una escala de cinco puntos $(1=$ Estoy completamente de acuerdo, 2 = Estoy parcialmente de acuerdo, 3 = Posición neutral, 4 = No estoy completamente de acuerdo y 5 = Estoy en total desacuerdo) que dan lugar a los seis tipos de amor: "Eros" (amor apasionado), "Ludus" (amor lúdico), "Storge" (amor basado en la amistad), "Pragma" (amor práctico), "Mania" (amor posesivo y dependiente) y "Agape" (amor generoso y desinteresado).

\section{Resultados}

Con el fin de investigar cómo variaban los síntomas del estrés traumático según el sexo, comparamos las puntuaciones medias obtenidas en la Escala que evalúa el impacto de los acontecimientos vitales (IES) y en el Cuestionario de Salud General (GHQ28). De acuerdo con la información que aporta la tabla II, aunque el grupo de mujeres tenía más "pensamientos intrusivos" sobre la ruptura, mostraban una actitud de rechazo mayor y habían obtenido una puntuación total superior a la de los hombres, los resultados no mostraban diferencias significativas entre ambos grupos.

En lo que a la intrusión respecta, al $28 \%$ de los hombres les venían con frecuencia a 
la mente imágenes sobre el final de la relación. Aseguraban que, a pesar de que intentaban evitarlo, pensaban con frecuencia en la ruptura (el 24\%). Otro $24 \%$ de estos hombres, con frecuencia cualquier cosa podía hacerles pensar en la ruptura y además, sus sentimientos podían verse afectados por estos recuerdos. El 16\% de los miembros de este grupo sufría crisis o episodios emocionales en relación con estos recuerdos. En cuanto a la evitación, el $24 \%$ intentó sentirse triste cuando pensaban en la ruptura. El $20 \%$ se sentía como si la relación no hubiese terminado y trató de no hablar sobre ello. El $16 \%$ procuró apartar de su memoria la ruptura de sus relaciones, aunque eran conscientes de que aún no habían logrado superar el problema.

En cuanto al grupo de las mujeres, en lo que a la intrusión respecta, el $28 \%$ afirmó que pensaban en la ruptura en cualquier momento del día. El 25\% sufría de episodios de episodios emocionales y recordaban el pasado con frecuencia. Aseguraban que cualquier acontecimiento servía para recordárselo. El 20\% pensaba en la ruptura habida, incluso cuando no era esa su intención. Respecto a la evitación, el 33\% trataba con frecuencia de apartar de su memoria la ruptura sufrida y el $25 \%$ evitaba todo aquello que pudiera recordársela, otro $25 \%$ no pensaba en ello y el $15 \%$ optó por no hablar del tema. El 23\% trataba de evitar sentirse triste cuando lo recordaba y el $15 \%$ era consciente de que aún no habían hecho frente a muchos de sus sentimientos. El 13\% intentaba sentir como que la ruptura no se había producido y otro $13 \%$ no sentía nada al respecto.

Basándonos en las recomendaciones de Horowitz sobre los síntomas IES altos medios y bajos, 44 personas (68\%) $(\mathrm{M}=14$, $\mathrm{F}=30$ ) obtuvieron la puntuación alta y para 21 personas $(32 \%),(\mathrm{M}=11, \mathrm{~F}=10) \operatorname{los}$ resultados fueron de tipo medio y bajo.

En cuanto a la salud en general (GHQ28), el grupo de mujeres obtuvo puntuaciones superiores a las del grupo de hombres en las cuatro subescalas y en la salud general total. Especialmente el grupo de mujeres mostraba más disfunciones sociales y depresiones y obtenía puntuaciones superiores en el GHQ-28 que los hombres. 29 individuos $(45 \%)(\mathrm{M}=8, \mathrm{~F}=21)$ fueron clasificados como casos psiquiátricos.

Se calcularon los coeficientes de correlación con el fin de establecer relaciones entre el impacto que produce la ruptura de una relación y el estado de salud general para ambos grupos. En cuanto a los hombres, los

Tabla II

La media y la desviación estándar de los IES y GHQ de los dos grupos

\begin{tabular}{lcrrrrr} 
& \multicolumn{2}{c}{ Hombres } & \multicolumn{2}{c}{ Mujeres } & $\mathrm{t}$ & $\mathrm{p}$ \\
\hline Items IES & Hombres & $\mathrm{SD}$ & Mujeres & $\mathrm{SD}$ & & \\
Intrusión & 13,88 & 9,25 & 15,77 & 9,65 & $-0,78$ & $\mathrm{~ns}$ \\
Evitación & 12,00 & 9,73 & 16,82 & 9,70 & $-1,94$ & $\mathrm{~ns}$ \\
Total & 25,88 & 16,71 & 32,60 & 16,45 & $-1,59$ & $\mathrm{~ns}$ \\
Items GHQ & & & & & & \\
Somático & 1,16 & 1,84 & 1,87 & 2,11 & $-1,39$ & $\mathrm{~ns}$ \\
Ansiedad & 1,56 & 2,27 & 2,30 & 2,44 & $-1,22$ & $\mathrm{~ns}$ \\
Desajuste social & 0,80 & 1,65 & 1,97 & 2,33 & $-2,36$ & 0,02 \\
Depresión & 0,48 & 1,08 & 1,67 & 2,39 & $-2,74$ & 0,01 \\
Total & 4,00 & 6,11 & 7,82 & 8,06 & $-2,16$ & 0,03 \\
\hline
\end{tabular}


"pensamientos intrusivos" sobre la ruptura de la relación, la consecuente actitud de evitación y el impacto total que supuso dicha ruptura estaban todos ellos relacionados con problemas somáticos, con la ansiedad con la disfunción social y con la depresión. De modo parecido, en el grupo de mujeres se descubrió que existían relaciones entre los pensamientos intrusivos sobre la ruptura de la relación, la actitud de rechazo con la consiguiente actitud de evitación y el impacto producido por la ruptura de la relación, además de la evitación, la disfunción social y los ítems relativos a la depresión (véase la tabla III).

Con respecto a la influencia que pudiera tener la pertenencia a un sexo determinado sobre la adopción de los estilos de amar, y a cómo las diferencias sexuales, el estrés traumático y los estilos de amar se pudieran influir mútuamente, se utilizó el Anova para comparar las puntuaciones medias de los estilos de amar según la gravedad del stress traumático (alto y bajo IES), el sexo de pertenencia y su interacción. Los resultados mostraron que existían diferencias entre ambos sexos en cuanto a la adopción de "Eros" $(\mathrm{F}=4,37, \mathrm{df}=1, \mathrm{p}<0,04) \mathrm{y}$ "Agape" ( $F=17,42$, dif $=1, p<0,001)$. Se encontraron también síntomas graves de estrés traumático en cuanto a los estilos de amar "Eros" ( $\mathrm{F}=4,40, \mathrm{df}=1, \mathrm{p}<0,04$ y "Mania" ( $F=12,40$, df $=1, p<0,01)$. Sin embargo, los resultados no mostraron ninguna interacción significativa entre el sexo, el nivel de gravedad del estrés traumático y los estilos de amor (véase la tabla IV).

Tabla III

Coeficientes de correlación entre el GHQ y el IES en ambos sexos

\begin{tabular}{lrrrrcc} 
& \multicolumn{3}{c}{ Hombres } & \multicolumn{2}{c}{ Mujeres } \\
\hline Items del GHQ & Intrusión & Evitación & IES total & Intrusión & Evitación & IES total \\
Somáticos & $0,68^{* *}$ & $0,47^{*}$ & $0,65^{* *}$ & $0,49^{* *}$ & $0,39 *$ & $0,52^{* *}$ \\
Ansiedad & $0,74^{* *}$ & $0,45^{*}$ & $0,67 * *$ & $0,45^{* *}$ & $0,41^{* *}$ & $0,50^{* *}$ \\
Desajuste social & $0,50^{* *}$ & $0,51^{* *}$ & $0,58^{* *}$ & $0,40^{*}$ & 0,26 & $0,38^{*}$ \\
Depresión & $0,44^{*}$ & 0,35 & $0,45^{*}$ & $0,34 *$ & 0,29 & $0,37^{*}$ \\
Total & $0,69 *$ & $0,51^{* *}$ & $0,68^{* *}$ & $0,48^{* *}$ & $0,39 *$ & $0,51^{* *}$ \\
\hline
\end{tabular}

(*) $\mathrm{p}<0,05$.

$(* *) \mathrm{p}<0,01$

Tabla IV

Puntuaciones de la actitud ante el amor según la gravedad de los síntomas de IES y el sexo

\begin{tabular}{lcccccccc} 
Estilos de amor & \multicolumn{2}{c}{ Síntomas altos en el IES } & \multicolumn{3}{c}{ Síntomas medio-bajos en el IES } \\
\hline & \multicolumn{2}{c}{ Hombres } & \multicolumn{2}{c}{ Mujeres } & \multicolumn{2}{c}{ Hombres } & \multicolumn{2}{c}{ Mujeres } \\
& Media & SD & Media & SD & Media & SD & Media & SD \\
\hline Eros & 13,14 & 3,88 & 16,90 & 6,08 & 16,90 & 4,32 & 19,00 & 4,13 \\
Ludus & 24,42 & 5,51 & 22,46 & 5,64 & 24,36 & 6,46 & 19,90 & 7,09 \\
Storge & 18,50 & 5,15 & 20,53 & 6,16 & 23,72 & 4,47 & 20,80 & 4,98 \\
Pragma & 27,78 & 6,93 & 27,90 & 5,22 & 28,81 & 5,92 & 27,90 & 4,20 \\
Mania & 19,50 & 3,58 & 21,23 & 7,54 & 28,00 & 5,74 & 25,20 & 6,97 \\
Agape & 12,35 & 3,05 & 19,60 & 6,31 & 16,09 & 6,64 & 21,50 & 4,45 \\
\hline
\end{tabular}




\section{Discusión}

La primera hipótesis de este estudio fue parcialmente confirmada por el hecho de que el grupo de mujeres tenía mayor disfunción social y se deprimían más que los hombres tras la ruptura de una relación. Sin embargo, las diferencias entre hombres y mujeres con respecto a los "pensamientos intrusivos" y a la evitación no fueron significativas a pesar de que ellas alcanzaron puntuaciones muy superiores a las de los hombres en el IES. Nuestros descubrimientos contradicen a las investigaciones comentadas, mostrando que ambos sexos sintieron angustia tras la ruptura de la relación con la misma intensidad (Frazier y Cook 1993, Helgeson 1994, Hortacsu y Karanci 1987, Simpson 1987). También ponen en duda a hallazgos anteriores, según los cuales los hombres se sentían más deprimidos e infelices que las mujeres tras la ruptura de una relación de este tipo (Hill, Rubin y Peplau 1976, Rubin, Peplau y Hill 1981) sin embargo confirman las teorías, según las cuales, las mujeres tienden a experimentar más desasosiego, angustia, y depresión grave que los hombres (Mearns 1991, Smith y Cohen 1993).

La correlación de los resultados revela claramente que para ambos sexos existe una relación significativa entre el impacto que provocó la ruptura de la relación y la salud en general. Así, tanto para los hombres como para las mujeres, cuanto mayor fue el impacto de la ruptura, peor era su estado de salud. Sin embargo, en ningún caso existía relación entre las actitudes de evitación y la depresión. En su conjunto, los resultados obtenidos confirman la creencia general de que la ruptura de una relación sentimental de este tipo es una experiencia traumática y estresante. Es importante mencionar que, según investigaciones previas, se produce un aumento de los casos de depresión (Sprecher 1994),de insomnio (Fink, Russell, Engel, Harkness, Pederson y Harvey 1991) y de enfermedades físicas (Williams y Siegel 1989), así como de sentimientos de indefensión y de preocupación ante el futuro (Harvey, Flanary y Morgan 1986), todos ellos también detectados por nosotros mediante el GHQ-28.

La diferencia en los estilos de amar entre hombres y mujeres fue mínima. El grupo de hombres tendía a adoptar los estilos "Eros" y "Agape" más que lo hacían las mujeres. Por lo demás, las actitudes ante el amor eran parecidas. Aunque estos resultados confirman algunos de los obtenidos en la bibliografía más reciente que señalan como los hombres tienden mas al estilo "Agape" que las mujeres (e.g. Hendrick y Hendrick 1986, Leon et al. 1995), en conjunto revelan pocas variaciones en los estilos de amar propios de ambos sexos.

Es importante señalar que estas conclusiones se deben interpretar teniendo en cuenta la metodología que se ha utilizado en este estudio. En primer lugar, las personas que participaron eran estudiantes universitarios. Todos ellos, probablemente pertenecían a una "sub-cultura" concreta, la "cultura de los estudiantes", compartiendo determinadas ideologías y formas de hacer las cosas, incluyendo en ello sus estilos de amar. Obviamente, no queremos decir que los estilos de amar sean idénticos para ambos grupos, sino que podrían recibir influencias emanantes de una misma ideología y cultura. Esto nos lleva al segundo punto, debiendo señalarse como la mayoría, sino todas las personas que participaron en el estudio eran de raza caucásica nacidas en Inglaterra, y adaptadas a la forma de vida y cultura inglesas. No nos sorprendió descubrir que las diferencias entre los estilos de amar fuesen pequeñas, ya que proba- 
blemente recibieron parecidas influencias de la sociedad en la que habían nacido y crecido.

En otras palabras, para descubrir más diferencias entre los estilos de amar de hombres y mujeres, necesitaríamos trabajar con una muestra más variada. Cabe preguntarse si el haber seleccionado a una población inglesa en la que se hubiesen incluido a personas de diferentes ambientes, hubiera afectado a las diferencias en los estilos de amar. Por ello sería muy positivo llevar a cabo un estudio intercultural mas profundo.

Estos descubrimientos no confirmaron las hipótesis, según las cuales existiría una interacción significativa entre los sexos, los estilos de amar y los síntomas de estrés postraumático producidos tras la ruptura de una relación. En otras palabras, mientras que las diferencias entre los sexos y la gravedad de los síntomas de estrés postraumático podrían ser responsables de forma independiente de la adopción de un forma de amar determinada, los resultados no serían iguales si ambos factores actuasen juntos. Si los estilos de amar fueran más variados, podríamos preguntarnos si la interacción entre el sexo, la gravedad de los síntomas de estrés postraumático y los estilos de amar hubieran sido más importantes.

Si quisiéramos ampliar la investigación en un futuro, en lugar de centrarnos exclusivamente en las diferencias entre hombres y mujeres, deberíamos estudiar también las diferencias individuales o la personalidad de cada individuo seleccionado. Esta información adicional nos ayudaría a descubrir algunos factores significativos que podrían interactuar con los estilos de amar. Por ejemplo, recientemente, hemos recogido datos sobre la personalidad de estudiantes universitarios que vivieron la ruptura de una relación siete meses aproximadamente antes de que se llevara a cabo este estudio. También se recogieron algunos datos sobre sus estilos de amar. Los resultados preliminares mostraron que estas personas tenían grados menores de psicoticismo, extroversión y neuroticismo. El psicoticismo y el neuroticismo estaban correlacionados con los estilos de amar "Eros" ( $\mathrm{r}=-0,29, \mathrm{p}<0,01)$ y "Mania" ( $\mathrm{r}=0,34, \mathrm{p}<0,01)$ respectivamente (Chung, Farmer, Grant, Newton, Payne, Perry, Saunders, Smith y Stone) (enviados para su publicación).

\section{Bibliografía}

BLOOM, B., ASHER, S.J., WHITE, S.W. Marital disruption as a stressor: A review and analysis. Psychological Bulletin, 85, 867-894, 1978.

CHUNG, M.C., FARMER, S., GRANT, K., NEWTON, R., PAYNE, S., PERRY, M., SAUNDERS, J., SMITH, C., STONE, N. Self-esteem, personality and post traumatic stress symptoms following the dissolution of a dating relationship. Submittod for publication.

FINK, K., RUSSELL, S., ENGEL, L., HARKNESS, D., PEDERSON, D., HARVEY, J. Reactions to relationships loss. Paper presented at Southwestern Psychological Association meeting. New Orleans, 1991.

FRAZIER, P.A., COOK, S.W. Correlates of distress following heterosexual relationship dissolution. Journal of Social and Personal Relationships, lO(1), 55-67, 1993.

GERRITY, E.T., STEINGLASS, P. Relocation stress following natural disasters. In R.J. Ursano, B.G. McCaughey., C.S. Fullerton (eds), Individual and Community Responses to Trauma and Disaster (pp. 220-247). Cambridge: Cambridge University Press, 1994.

GLASS, S.P., WRIGHT, T.L. Reconstructing marriages atter the trauma of infidelity. In W.K. Halford, H.J. Markman (eds), Clinical handbook of marriage and couples interventions (pp. 471-507). New York: John Wiley \& Sons Ltd, 1997.

GOLDBERG, D.P., HILLIER, V.F. A scaled version of the General Health Questionnaire. Psychological Medicine, 9, 139-145, 1979. 
HALL, A.G., HENDRICK, S.S., HENDRICK, C. Personal construct systems and love styles. International Journal of Personal Construct Psychology, 4, 137-155, 1991.

HARVEY, J.H., FLANARY, R., MORGAN, M. Vivid memories of vivid loves gone by. Journal of Social and Personal Relationships, 3, 359-373, 1986.

HELGESON, V.S. The effects of self-beliefs and relationship beliefs on adjustment to a relationship stressor. Personal Relationships, 1(3), 241-258, 1994.

HENDRICK, C., HENDRICK, S.S. A theory and method of love. Journal of Personality and Social Psychology, 50, 392-402, 1986.

HENDRICK, S.S., HENDRICK, C. Love and sex attitudes and religious beliefs. Journal of Social and Clinical Psychology, Vol 5(3), 391-398, 1987.

HENDRICK, C., HENDRICK, S.S. A relationship-specific version of the Love Attitudes Scale. Journal of Social Behavior and Personality, 5, 239-254, 1990.

HILL, C.T., RUBIN, ZICK., PEPLAU, L.A. Breakups before marriage: The end of 103 affairs. Journal of Social Issues, 32(1), 147-168, 1976.

HONG, S.M. Romantic love, idealistic or pragmatic: Sex differences among Australian young adults. Psychological Reports, Vol 58(3): 922, 1986.

HOROWITZ, M., WILNER, N., ÁLVAREZ, W. Impact of Events Scale: A measure of subjective stress. Psychosomatic Medicine, 41 (3), 209-218, 1979.

HORTACSU, N., KARANCI, A.N. Premarital breakups in a Turkish sample: Perceived reasons, attributional dimensions and affective reactions. International Journal of Psychology, 22(1), 57-74, 1987.

HUANG, M.H. Cross-cultural similarity in the Love Attitudes Scale: Short Form. Psychological Reports, Vol 84(2), 617-624, 1999.

LAGRAND, L.E. Changing patterns of human existence: Assumptions, beliefs, and coping with the stress of change. Springfield: Charles C. Thomas Publisher, 1988.

LEE, J.A. The colors of love: An exploration of the ways of loving. Don Mills: New Press, 1973.

LEE, J.A. Love-styles. In R.J. Sternberg, M.L. Barnes (eds.). The psychology of love (pp. 38-67). New Haven: Yale University Press, 1988.

LEON, J.J., PARRA, F., CHENG, T., FLORES, R.E. Love-styles among Latino community colloge students in Los Angeles. Psychological Reports, Vol 77(2), 527-530, 1995.
LORENZ, F.O., SIMONS, R.L., CHAO, W. Family structure and mother's depression. In R.L. Simons (eds), Understanding differences between divorced and intact families: Stress, interaction, and child outcome (pp. 6577). Thousand Oaks: Sage Publications, Inc., 1996.

LUSTERMAN, D. Treating marital infidelity. In R.H. Mikesell., D. Lusterman., S.H. McDaniel (eds), Integrating family therapy: Handbook of family psychology and systems theory (pp. 259-270). Washington: American Psychological Association, 1995.

MASTEKAASA, A. Marital dissolution as a stressor: Some evidence on psychological, physical, and behavioral changes in the pre-separation period. Journal of Divorce and Remarriage, 26(3-4), 155-183, 1997.

MASTEKAASA, A. Psychological well-being and marital dissolution: Selection effects? Journal of Family Issues, 15(2), 208-228, 1994.

MEARNS, J. Coping with a breakup: Negative mood regulation expectancies and depression following the end of a romantic relationship. Journal of Personality and Social Psychology, 60(2), 327-334, 1991.

MENAGHAN, E.G., LIEBERMAN, M.A. Changes in depression following divorce: A panel study. Journal of Marriage and the Family, 48, 319-328, 1986.

MITCHELL, M. The aftermath of road accidents. London: Routledge, 1997.

MURSTEIN, B.I. MERIGHI, J.R., VYSE, S.A. Love styles in the United States and France: A cross-cultural comparison. Journal of Social and Clinical Psychology, 10, 37-46, 1991.

ROTENBERG, K.J., KOROL, S. The role of loneliness and gender in individuals' love styles. Journal of Social Behavior and Personality, Vol 10(3), 537-546, 1995.

RUBIN, Z., PEPLAU, L.A., HILL, C.T. Loving and leaving: Sex differences in romantic attachments. Sex Roles, 7, 821-835, 1981.

SIMPSON, J.A. The dissolution of romantic relationships: Factors involved in relationship stability and emotional distress. Journal of Personality and Social Psychology, 53(4), 683-692, 1987.

SMITH, H.S., COHEN, L.H. Self-complexity and reactions to a relationship breakup. Journal of Social and Clinical Psychology, 12(4), 367-384, 1993.

SPRECHER, S. Two sides to the breakup of dating relationships. Personal Relationships, 1(3), 199-222, 1994.

STROEBE, W., STROEBE, M.S. Beyond marriage: The impact of partner loss on health. In R. Gilmour., S. 
Duck (eds), The emerging field of personal relatioinship (pp. 203-224). Hillsdale, NJ: Erlbaum, 1986.

WEISAETH, L. Psychological and psychiatric aspects of technological disasters. In R.J. Ursano., B.G. McCaughey., C.S. Fullerton (eds) Individual and Community Responses to Trauma and Disaster (pp. 72-102). Cambridge: Cambridge University Press, 1994.

WILLIAMS, J.S., SIEGEL, J.P. Marital disruption and physical illness: The impact of divorce and spouse death on illness. Journal of Traumatic Stress, 2(4), 555-562, 1989.
Dirección para correspondencia:

Dr. M.C. Chung

Department of Psychology

University of Plymouth

Drake Circus

Plymouth, Devon

PL4 8AA

REINO UNIDO 